\title{
The likelihood of total knee arthroplasty following arthroscopic surgery for osteoarthritis: a systematic review
}

\author{
Amelia R. Winter ${ }^{1}$, Jamie E. Collins ${ }^{1,3}$ and Jeffrey N. Katz ${ }^{1,2,3,4^{*}}$
}

\begin{abstract}
Background: Arthroscopic surgery is a common treatment for knee osteoarthritis (OA), particularly for symptomatic meniscal tear. Many patients with knee OA who have arthroscopies go on to have total knee arthroplasty (TKA). Several individual studies have investigated the interval between knee arthroscopy and TKA. Our objective was to summarize published literature on the risk of TKA following knee arthroscopy, the duration between arthroscopy and TKA, and risk factors for TKA following knee arthroscopy.

Methods: We searched PubMed, Embase, and Web of Science for English language manuscripts reporting TKA following arthroscopy for knee OA. We identified 511 manuscripts, of which 20 met the inclusion criteria and were used for analysis. We compared the cumulative incidence of TKA following arthroscopy in each study arm, stratifying by type of data source (registry vs. clinical), and whether the study was limited to older patients $(\geq 50)$ or those with more severe radiographic OA. We estimated cumulative incidence of TKA following arthroscopy by dividing the number of TKAs among persons who underwent arthroscopy by the number of persons who underwent arthroscopy. Annual incidence was calculated by dividing cumulative incidence by the mean years of follow-up.

Results: Overall, the annual incidence of TKA after arthroscopic surgery for OA was 2.46\% (95\% Cl 1.68-3.25\%). We calculated the annual incidence of TKA following arthroscopy in four separate groups defined by data source (registry vs. clinical cohort) and whether the sample was selected for disease progression (either age or OA severity). In unselected registry studies the annual TKA incidence was 1.99\% (95\% Cl 1.03-2.96\%), compared to 3.89\% (95\% Cl 0.69-7.09\%) in registry studies of older patients. In unselected clinical cohorts the annual incidence was $2.02 \%$ (95\% Cl 0.67-3.36\%), while in clinical cohorts with more severe OA the annual incidence was 3.36\% (95\% Cl 1.38-5.34\%). The mean and median duration between arthroscopy and TKA (years) were 3.4 and 2.0 years.
\end{abstract}

Conclusions: Clinicians and patients considering knee arthroscopy should discuss the likelihood of subsequent TKA as they weigh risks and benefits of surgery. Patients who are older or have more severe OA are at particularly high risk of TKA.

Keywords: Osteoarthritis, Total knee arthroplasty, Arthroscopic partial meniscectomy, Arthroscopy

\section{Background}

Osteoarthritis $(\mathrm{OA})$ is a debilitating disease, affecting over 40 million people in the United States [1,2]. Of those affected, approximately 14 million have symptomatic knee OA [3], which presents with pain, loss of knee joint function, and loss of valued activities. In addition, about $90 \%$ of

\footnotetext{
* Correspondence: jnkatz@partners.org

'Orthopaedic and Arthritis Center for Outcomes Research (OrACORe),

Department of Orthopedic Surgery, Boston, MA, USA

2Division of Rheumatology, Immunology and Allergy, Brigham and Women's

Hospital, 60 Fenwood St, Suite 5016, Boston, MA 02115, USA

Full list of author information is available at the end of the article
}

those with symptomatic knee OA have meniscal tears (MT) documented on magnetic resonance imaging (MRI) [4]. However, no available treatments modify the structural progression associated with OA. Symptoms are generally managed with conservative therapies (e.g., nonsteroidal anti-inflammatory drugs (NSAIDs), exercise, physical therapy). Patients and their physicians often turn to surgical treatments to address progressive pain and disability, including arthroscopy and total knee arthroplasty (TKA).

Over 600,000 arthroscopic partial meniscectomies (APM) are performed each year in the United States [5], 
most commonly on persons over 45 with MT $[5,6]$. The benefit of arthroscopic surgery in patients with OA is uncertain and debated. Moseley et al. showed that sham surgery and arthroscopic surgery for OA had similar pain relief and functional improvement up to 2 years post-surgery [7]. Kirkley and colleagues showed that arthroscopy and a conservative exercise regimen had similar symptomatic and functional outcomes in persons with knee OA [8]. With respect to MT in the setting of OA, several trials demonstrated that surgery was not superior to nonoperative therapy or sham surgery in intention to treat analyses [9-12], while one trial showed a benefit for surgery [13]. Thus, arthroscopic surgery is felt to be ineffective for OA per se, while the effectiveness of APM in persons with MT and concomitant OA is debated [14].

Often, people who undergo arthroscopic surgery for osteoarthritis progress to TKA. While some studies suggest that up to $20 \%$ of patients undergo TKA within one year of arthroscopy [15], other studies have shown TKA rates under 5\% [16]. The various studies of the rate of TKA after arthroscopy have not been summarized, to our knowledge. Such a summary of the risk of TKA following arthroscopy and the duration between arthroscopy and TKA would be helpful for clinicians to better advise patients and their families on appropriate treatments plans. Surgery is expensive - about $\$ 2$ billion dollars are spent on arthroscopy for OA [17] and over $\$ 10$ billion dollars are spent on TKAs each year [18]. Therefore, improved knowledge of the risk for TKA following arthroscopy could also lead to better resource allocation for OA.

We performed a systematic review of the literature on the risk of TKA following arthroscopic surgeries for OA. We expected to see older patients and those with more severe OA, progress to TKA more quickly after surgery.

\section{Methods}

\section{Definition of search terms}

A search by title was performed on PubMed, Embase, and Web of Science using the major search terms: osteoarthritis, knee; arthroscopy; and arthroplasty (see Additional file 1: Table S1 for search strings). The search was performed in September 2016 and titles were downloaded to EndNote. One reviewer (ARW) manually screened titles for inclusion and exclusion criteria, arriving at a final list of titles. For these titles, the reviewer assessed abstracts for inclusion and exclusion criteria. For each abstract that was not excluded, the full manuscript was read to determine ultimate inclusion in the final analysis. A second reviewer confirmed that the final selected manuscripts met inclusion criteria.

\section{Inclusion and exclusion criteria}

We sought studies investigating the rate of arthroplasty after arthroscopic knee surgery for osteoarthritis. Therefore, inclusion criteria included: English language and human studies on the risk of TKA occurring following arthroscopic procedures for knee OA. Manuscripts were excluded if they were duplicates, written in a language other than English, or conducted on animals. Studies were also excluded if they examined only arthroscopy or arthroplasty rather than the risk of arthroplasty following arthroscopy. Case studies and studies with cohorts of mean age $<40$ were also excluded. Confusion regarding inclusion of a study was resolved by consulting with the senior author (JNK).

\section{Data abstraction}

From the manuscripts, the reviewer (ARW) extracted the following information (if available): author, year, title, administrative data (e.g., clinical cohort or registry), country, patient selection criteria (e.g. age, KL grade), subgroup information, size of analysis group, mean age, analysis method (e.g., cumulative incidence), duration between arthroscopy and TKA, duration of follow-up, percentage of TKA, and study arm population description. Another reviewer abstracted key data (e.g., country, administrative data, patient selection criteria, follow-up years, analysis group, and total TKA numbers) from the included studies. The results from both abstractions were compared and found to be the same.

\section{Categorization of studies}

We examined the cumulative incidence of TKA following knee arthroscopy in specific patient subgroups. These included source of data (administrative data registries vs. clinical cohort studies), OA severity, older age (e.g., selection for population $\geq 50$ ), and country. Some of the clinical cohort studies recruited patients with advanced OA, (i.e., KL grade $\geq 3$ or Outerbridge score $\geq 2$ ). We defined these study arms as "Clinical Cohort - More Severe OA." One study (Lyu et al., 2015) was included among the "Clinical Cohort - More Severe OA" group as its patient population was over $75 \%$ KL grade 3 or higher. Some registry studies were restricted to subjects with age greater than 50. We referred to these as "Registry - Older Age." We created a final categorization combining the source of data and selection criteria: "Registry - Unselected," "Registry - Older Age," "Clinical Cohort - Unselected," and "Clinical Cohort - More Severe OA." For countries, we combined England and Scotland as "U.K."

\section{Quality assessment}

We used the Quality Assessment Tool for Observational Cohort and Cross-Sectional Studies developed by investigators at the National Heartt, Lung and Blood Intitute (NHLBI), based upon work done at the Agency for Health Care Research and Quality. (https://www.nhlbi.nih.gov/ health-pro/guidelines/in-develop/cardiovascular-risk-reduct ion/tools/cohort) [19]. This measure includes 14 items 
relevant to the quality of cohort studies, with emphasis on explicit specification of sample characteristics, exposures, primary outcomes and potential confounders. Two authors performed the assessment independently and resolved any disagreements.

\section{Analyses}

For studies that did not provide cumulative incidence data, we calculated cumulative incidence using the number of TKAs divided by the number of arthroscopic patients included for follow-up analysis. We first examined the association between the type of data source - registry vs. cohort - and TKA rates. Then, we evaluated the association between study category ("Registry - Unselected," "Registry - Older Age," "Clinical Cohort - Unselected," and "Clinical Cohort - More Severe OA," as described above) and annual incidence. In secondary analyses, we evaluated the difference between TKA annual incidence in unselected study arms (clinical and registry) vs. selected study arms, regardless of registry status. We compared studies in which mean age of the study was $>65$ to those with mean age $<65$.

We divided cumulative incidence of TKA by mean years of follow-up to obtain an annual incidence estimate. We computed exact confidence intervals for each yearly incidence value. We used a logistic random-effects model to create an overall combined estimate of annual TKA incidence across all studies and to evaluate the effect of study-level characteristics on TKA incidence. This approach allows for studies with zero cells (i.e., $0 \%$ incidence rate) without requiring an ad-hoc adjustment [20]. All analyses were conducted using SAS 9.4 (SAS Institute, Cary NC).

\section{Results}

Five hundred eleven unique articles were found using our search terms and three search engines. After screening the titles, we were left with 328 articles whose abstracts were subsequently reviewed. Over half of the articles excluded did not have arthroscopic surgery before TKA (36\%) or did not report TKA (24\%). Fifty-five articles underwent full article review. Thirty-five of the manuscripts were excluded from our analysis: 11 were not written in English, 2 did not report on arthroscopic procedures before TKA, 3 did not report on TKA, and 19 were excluded for other reasons, such as mixed cohort (e.g., OA and post-traumatic arthritis), secondary sources, or insufficient data on methodology.

These exclusions left 20 articles for the analysis (Fig. 1). The 20 studies contained 28 unique study arms (Table 1). The 28 study arms were reported from eight countries. The U.S.A. accounted for 15 of the 28, the U.K. for 5 , Canada for 3, and Australia, Belgium, Italy, South Korea, and Taiwan for one each. The quality assessment documented relatively little variability in quality. Essentially all the studies stated the research question clearly, specified the population and defined the exposure and outcome explicitly. Only one study provided a power calculation. Rates of participation among eligible subject and rates of follow up were generally high, particularly for administrative data studies in which participation and follow-up rates are typically $100 \%$. Some of the quality items did not apply to the studies we reviewed because all subjects in our studies were 'exposed' (had arthroscopy).

Overall, the yearly incidence for TKA after arthroscopic surgery for OA was $2.46 \%$ (95\% CI 1.68-3.25\%). The mean and median duration between arthroscopy and TKA (years) were 3.4 and 2.0 years. From our 28 study arms, we identified sixteen clinical cohorts and twelve registry samples. The clinical cohort studies had a yearly TKA incidence of $2.60 \%$ (95\% CI $1.38-3.81 \%$ ), compared to the registry studies, which had an incidence of $2.36 \%$ (95\% CI 1.26-3.46\%) $(p=0.7591)$. We examined separately the risk of TKA in four distinct subgroups: "Registry - Unselected," "Registry - Older Age," "Clinical Cohort - Unselected," and "Clinical Cohort - More Severe OA." The four subgroups are shown in Fig. 2.

\section{Registry - unselected}

A total of nine study arms were unselected registries, with a median of 6972 (range 842-159,975) patients per study arm (Table 1). Of these, the average yearly incidence for TKA was 1.99\% (95\% CI 1.03-2.96\%) (Fig. 2).

\section{Registry - Older age}

A total of three study arms were registries using data from patients $\geq 50$ years old, with a median of 6212 (range 3033-40,804) patients per study arm (Table 1). Of these studies, the average yearly incidence for TKA was 3.89\% (95\% CI 0.69-7.09\%) (Fig. 2).

\section{Clinical cohort - Unselected}

A total of seven study arms were unselected clinical cohorts, with a median of 42 (range 8-183) patients per study arm (Table 1). Of these studies, the average yearly incidence for TKA was $2.02 \%$ (95\% CI 0.67-3.36\%) (Fig. 2).

\section{Clinical cohort - More severe OA}

A total of nine study arms were clinical cohorts selecting for patients with more severe OA on the basis of KL grade or Outerbridge score, with a median of 69 (range 68-844) patients per study arm (Table 1). Of these studies, the average yearly incidence for TKA was 3.36\% (95\% CI 1.38\%-5.34\%) (Fig. 2).

\section{Comparisons: Age and OA severity}

We evaluated the association between TKA incidence and study inclusion criteria using a logistic randomeffects model. We found that selected studies - those 


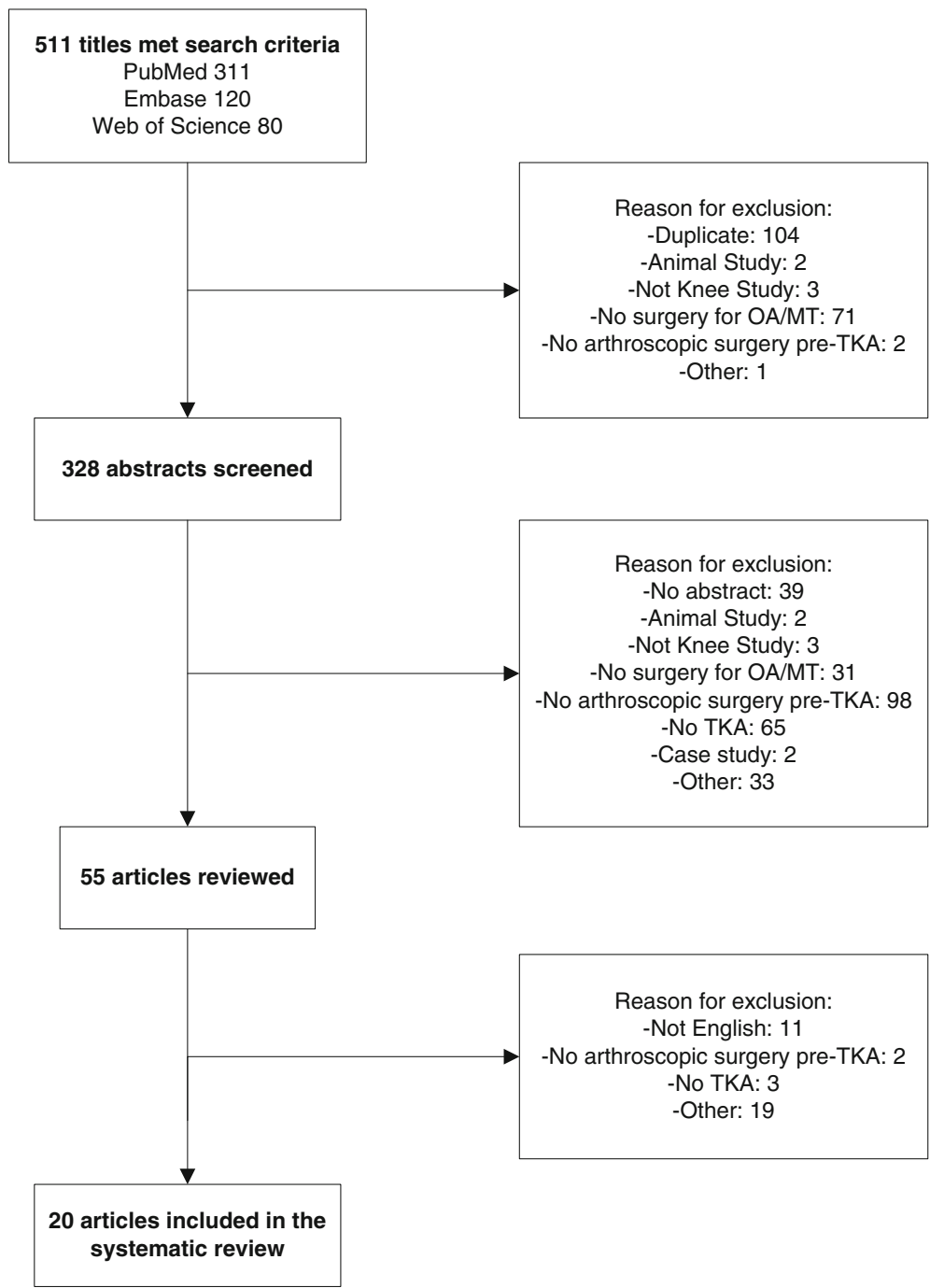

Fig. 1 Search and selection process

that selected subjects based on OA severity or age were twice as likely to undergo TKA compared to unselected studies (3.54\% compared to $2.00 \% ; p=0.0626$ ). Studies of subject with a mean age of less than 65 had a yearly incidence of $1.87 \%$ (95\% CI 1.16-2.57\%) compared to $5.13 \%$ (95\% CI $2.61-7.64 \%$ ) for those with mean age over 65 . This difference was statistically significant $(p=0.0027)$.

\section{Discussion}

We evaluated published literature on the risk of TKA in patients undergoing knee arthroscopy. A concern about the use of arthroscopic surgery in the setting of OA and OA with meniscal tear is that APM may lead to more rapid OA progression, leading to TKA more quickly $[1,15]$. We found that on average the risk of TKA following arthroscopy was about $2 \%$ per year and that the mean and medican duration between arthroscopy and TKA were 3.4 and 2.0 years respectively. Further, study arms of patients who were older or had more advanced radiographic OA at the time of arthroscopy had two-fold higher risk of TKA than unselected study arms. These findings should be viewed in the context of other documented risk factors for OA progression including older age, female gender, varus and valgus malalignment and bone marrow lesions, among others [21].

Our findings are consistent with studies showing that OA severity and age are associated with TKA [22-32]. Indeed, surgeons may be reluctant to offer TKA to younger patients, because they face a risk of a revision TKA. Advanced OA is a typical indication for TKA, as embodied in guidelines such as those of the American Academy of Orthopaedic Surgeons [33].

Our study must be interpreted in the context of several limitations. The clinical cohort data provided insight into the KL grades and Outerbridge scores of patients 


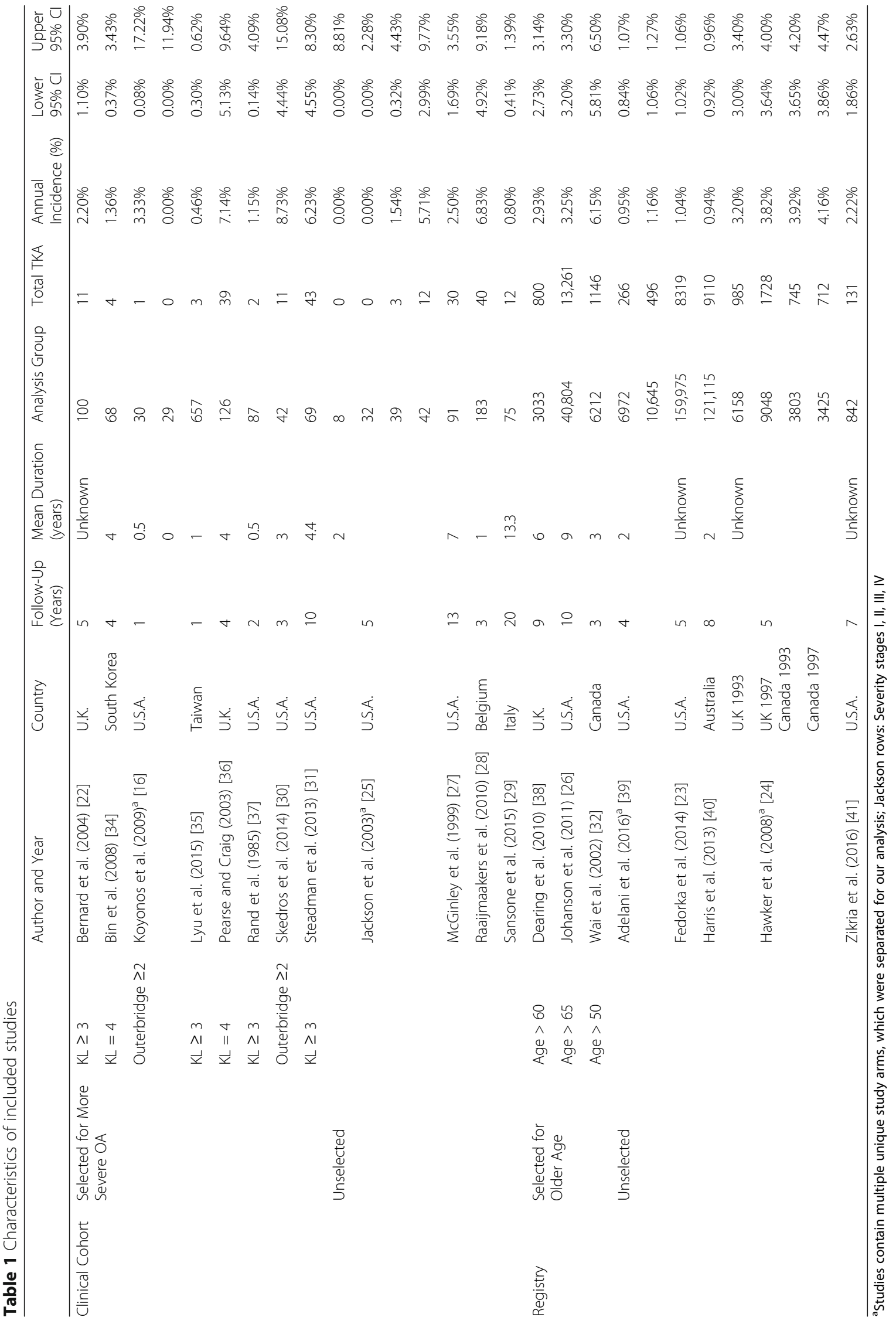




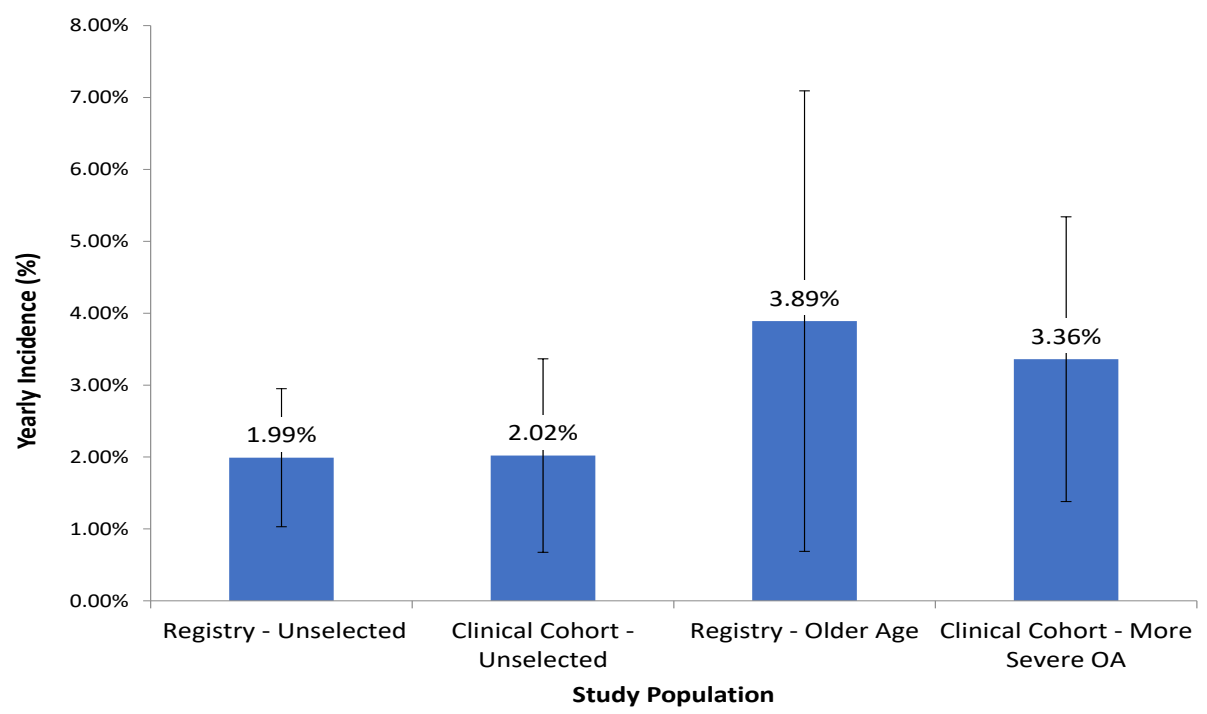

Fig. 2 Mean Annual Incidence of Registry - Unselected, Clinical Cohort - Unselected, Registry - Older Age, and Clinical Cohort - More Severe OA. Each bar represents the estimated yearly incidence of TKA from the logisitc random effects model. The vertical lines represent the $95 \%$ confidence intervals

whereas registry data included age but no information on OA severity nor on the details of surgery. The component studies did not perform analyses of subgroups that might be prognostically distinct, such as athletes and non-athletes, or males and females. Similarly, over half of the countries contributed just one cohort. This precludes meaningful analysis of between-country differences. While we performed replicate abstractions of all papers we did not repeat the screening of titles and abstracts in duplicate, creating the theoretical risk of our missing an eligible paper. As reflected in our quality assessment, the studies consistently defined the exposure and outcome explicitly. Since most of the larger studies used administrative data, the follow-up rates were generally $100 \%$. We note as well that some patients with a medical 'need' for TKA (symptomatic, advanced OA) may not have received the procedure because of their own preferences or the practice styles of their physicians or still other reasons. When TKA is used as a health outcome, the role of these patient, physician and health system factors may attenuate the risk associated with specific variables such as prior arthroscopic surgery.

To the best of our knowledge, this is the first systematic review to analyze the yearly TKA incidence rate for those having undergone arthroscopic surgery for knee OA. Quality assessment of the studies generally reflected consistent specification of exposures, outcomes, and study samples and high rates of participation and follow-up. The findings suggest that OA patients undergoing arthroscopy and their physicians should anticipate an annual rate of TKA on the order of $2 \%$, with higher rates among older patients and those with more advanced OA. These findings should be shared with patients when clinicians discuss the advantages and drawbacks of arthroscopy.

\section{Conclusion}

Clinicians and patients considering knee arthroscopy should discuss the likelihood of subsequent TKA as they weigh risks and benefits of surgery. Patients who are older or have more severe OA are at particularly high risk of TKA.

\section{Additional file}

Additional file 1: Table S1. Search Strings by Search Engines. Table of bibliographic search terms used to identify candidate articles. (DOCX $13 \mathrm{~kb}$ )

\section{Abbreviations}

KL: Kellgren-Lawrence; MRI: Magnetic resonance imaging; MT: Meniscal tear; NSAIDs: Nonsteroidal anti-inflammatory drugs; OA: Osteoarthritis; tka: Total knee arthroplasty

\section{Acknowledgements}

Not applicable.

\section{Funding}

National Institute of Arthritis, Musculoskeletal and Skin Diseases (National Institutes of Health) R01 AR 055557.

Availability of data and materials

All data generated or analysed during this study are included in this published article [and its Additional file 1].

\section{Authors' contributions}

ARW and JNK developed final question and design of systematic review. ARW searched for and analyzed the included manuscripts, and was a major contributor to writing the manuscript. JEC performed the analysis in SAS, discussed the interpretation with the other authors and critically reviewed the manuscript. JNK consulted on final included manuscripts. All authors read, edited, and approved the final manuscript. 


\section{Ethics approval and consent to participate}

Not applicable.

\section{Consent for publication}

Not applicable.

\section{Competing interests}

The authors declare they have no competing interests.

\section{Publisher's Note}

Springer Nature remains neutral with regard to jurisdictional claims in published maps and institutional affiliations.

\section{Author details}

Orthopaedic and Arthritis Center for Outcomes Research (OrACORe), Department of Orthopedic Surgery, Boston, MA, USA. ${ }^{2}$ Division of Rheumatology, Immunology and Allergy, Brigham and Women's Hospital, 60 Fenwood St, Suite 5016, Boston, MA 02115, USA. ${ }^{3}$ Harvard Medical School, Boston, MA, USA. ${ }^{4}$ Departments of Epidemiology and Environmental Health, Harvard T. H. Chan School of Public Health, Boston, MA, USA.

Received: 4 May 2017 Accepted: 21 September 2017

Published online: 04 October 2017

\section{References}

1. Choong PF, Dowsey MM. Update in surgery for osteoarthritis of the knee. Int J Rheum Dis. 2011;14(2):167-74.

2. Dunlop DD, Manheim LM, Yelin EH, Song J, Chang RW. The costs of arthritis. Arthritis Rheum. 2003:49(1):101-13.

3. Deshpande BR, Katz JN, Solomon DH, Yelin EH, Hunter DJ, Messier SP, Suter LG, Losina E. Number of persons with symptomatic knee osteoarthritis in the US: impact of race and ethnicity, age, sex, and obesity. Arthritis Care Res. 2016:68(12):1743-50.

4. Bhattacharyya T, Gale D, Dewire P, Totterman S, Gale ME, McLaughlin S, Einhorn TA, Felson DT. The clinical importance of meniscal tears demonstrated by magnetic resonance imaging in osteoarthritis of the knee. J Bone Joint Surg Am. 2003;85-A(1):4-9.

5. Cullen KA, Hall MJ, Golosinskiy A. Ambulatory surgery in the United States. Natl Health Stat Rep. 2006;2009(11):1-25.

6. Kim S, Bosque J, Meehan JP, Jamali A, Marder R. Increase in outpatient knee arthroscopy in the United States: a comparison of National Surveys of Ambulatory Surgery, 1996 and 2006. J Bone Joint Surg Am. 2011;93(11):994-1000.

7. Moseley JB, O'Malley K, Petersen NJ, Menke TJ, Brody BA, Kuykendall DH Hollingsworth JC, Ashton CM, Wray NP. A controlled trial of arthroscopic surgery for osteoarthritis of the knee. N Engl J Med. 2002;347(2):81-8.

8. Kirkley A, Birmingham TB, Litchfield RB, Giffin JR, Willits KR, Wong CJ, Feagan BG, Donner A, Griffin SH, D'Ascanio LM, et al. A randomized trial of arthroscopic surgery for osteoarthritis of the knee. N Engl J Med. 2008; 359(11):1097-107.

9. Herrlin SV, Wange PO, Lapidus G, Hallander M, Werner S, Weidenhielm L. Is arthroscopic surgery beneficial in treating non-traumatic, degenerative medial meniscal tears? A five year follow-up. Knee Surg Sports Traumatol Arthrosc. 2013;21(2):358-64.

10. Katz JN, Brophy RH, Chaisson CE, de Chaves L, Cole BJ, Dahm DL, Donnell-Fink LA, Guermazi A, Haas AK, Jones MH, et al. Surgery versus physical therapy for a meniscal tear and osteoarthritis. N Engl J Med. 2013;368(18):1675-84.

11. Sihvonen $\mathrm{R}$, Paavola M, Malmivaara A, Itala A, Joukainen A, Nurmi $H$, Kalske J, Jarvinen TL. Arthroscopic partial meniscectomy versus sham surgery for a degenerative meniscal tear. N Engl J Med. 2013;369(26):2515-24.

12. Yim JH, Seon JK, Song EK, Choi Jl, Kim MC, Lee KB, Seo HY. A comparative study of meniscectomy and nonoperative treatment for degenerative horizontal tears of the medial meniscus. Am J Sports Med. 2013;41(7):1565-70.

13. Gauffin H, Tagesson S, Meunier A, Magnusson H, Kvist J. Knee arthroscopic surgery is beneficial to middle-aged patients with meniscal symptoms: a prospective, randomised, single-blinded study. Osteoarthr Cartil. 2014;22(11): 1808-16.

14. Katz JN, Jones MH. Treatment of Meniscal Tear: the more we learn, the less we knowtreatment of meniscal tear: the more we learn, the less we know. Ann Intern Med. 2016;164(7):503-4.
15. Dervin GF, Stiell IG, Rody K, Grabowski J. Effect of arthroscopic debridement for osteoarthritis of the knee on health-related quality of life. J Bone Joint Surg Am. 2003;85-A(1):10-9.

16. Koyonos L, Yanke AB, McNickle AG, Kirk SS, Kang RW, Lewis PB, Cole BJ. A randomized, prospective, double-blind study to investigate the effectiveness of adding DepoMedrol to a local anesthetic injection in postmeniscectomy patients with osteoarthritis of the knee. Am J Sports Med. 2009;37(6):1077-82.

17. Losina E, Dervan EE, Paltiel AD, Dong Y, Wright RJ, Spindler KP, Mandl LA, Jones MH, Marx RG, Safran-Norton CE, et al. Defining the value of future research to identify the preferred treatment of meniscal tear in the presence of knee osteoarthritis. PLoS One. 2015;10(6):e0130256.

18. Healthcare Cost and Utilization Project. Nationwide Inpatient Sample. Rockville: Agency for Healthcare Research and Quality; 2014.

19. Quality Assessment Tool for Observational Cohort and Cross-Sectional Studies. [https://www.nhlbi.nih.gov/health-pro/guidelines/in-develop/ cardiovascular-risk-reduction/tools/cohort]

20. Hamza TH, van Houwelingen HC, Stijnen T. The binomial distribution of meta-analysis was preferred to model within-study variability. J Clin Epidemiol. 2008;61(1):41-51.

21. Hunter DJ. Risk stratification for knee osteoarthritis progression: a narrative review. Osteoarthr Cartil. 2009:17(11):1402-7.

22. Bernard J, Lemon M, Patterson MH. Arthroscopic washout of the knee-a 5year survival analysis. Knee. 2004;11(3):233-5.

23. Fedorka CJ, Cerynik DL, Tauberg B, Toossi N, Johanson NA. The relationship between knee arthroscopy and arthroplasty in patients under 65 years of age. J Arthroplast. 2014;29(2):335-8.

24. Hawker G, Guan J, Judge A, Dieppe P. Knee arthroscopy in England and Ontario: patterns of use, changes over time, and relationship to total knee replacement. J Bone Joint Surg Am. 2008;90(11):2337-45.

25. Jackson RW, Dieterichs $C$. The results of arthroscopic lavage and debridement of osteoarthritic knees based on the severity of degeneration: a 4- to 6-year symptomatic follow-up. Arthroscopy. 2003;19(1):13-20.

26. Johanson NA, Kleinbart FA, Cerynik DL, Brey JM, Ong KL, Kurtz SM. Temporal relationship between knee arthroscopy and arthroplasty. a quality measure for joint care? J Arthroplast. 2011;26(2):187-91.

27. McGinley BJ, Cushner FD, Scott WN: Debridement arthroscopy. 10-year followup. Clin Orthop Relat Res 1999(367):190-194.

28. Raaijmaakers M, Vanlauwe J, Vandenneucker H, Dujardin J, Bellemans J. Arthroscopy of the knee in elderly patients: cartilage lesions and their influence on short term outcome. A retrospective follow-up of 183 patients. Acta Orthop Belg. 2010;76(1):79-85.

29. Sansone V, de Girolamo L, Pascale W, Melato M, Pascale V. Long-term results of abrasion arthroplasty for full-thickness cartilage lesions of the medial femoral condyle. Arthroscopy. 2015;31(3):396-403.

30. Skedros JG, Knight AN, Thomas SC, Paluso AM, Bertin KC. Dilemma of high rate of conversion from knee arthroscopy to total knee arthroplasty. Am J Orthop (Belle Mead NJ). 2014:43(7):E153-8.

31. Steadman JR, Briggs KK, Matheny LM, Ellis HB. Ten-year survivorship after knee arthroscopy in patients with Kellgren-Lawrence grade 3 and grade 4 osteoarthritis of the knee. Arthroscopy. 2013;29(2):220-5.

32. Wai EK, Kreder HJ, Williams JI. Arthroscopic débridement of the knee for osteoarthritis in patients fifty years of age or older: Utilization and outcomes in the province of Ontario. Journal of Bone and Joint Surgery - Series A. 2002; 84(1):17-22+Adv26

33. Total Knee Replacement. American Academy of Orthopaedic Surgeons. 2015. http://orthoinfo.aaos.org/topic.cfm?topic=a00389. Accessed 4 April 2017

34. Bin SI, Lee SH, Kim CW, Kim TH, Lee DH. Results of arthroscopic medial meniscectomy in patients with grade IV osteoarthritis of the medial compartment. Arthroscopy. 2008;24(3):264-8.

35. Lyu SR. Knee health promotion option for knee osteoarthritis: A preliminary report of a concept of multidisciplinary management. Healthy Aging Research. 2015;4

36. Pearse EO, Craig DM. Partial meniscectomy in the presence of severe osteoarthritis does not hasten the symptomatic progression of osteoarthritis. Arthroscopy. 2003;19(9):963-8.

37. Rand JA. Arthroscopic management of degenerative meniscus tears in patients with degenerative arthritis. Arthroscopy. 1985:1(4):253-8.

38. Dearing J, Brenkel IJ. Incidence of knee arthroscopy in patients over 60 years of age in Scotland. The surgeon: journal of the Royal Colleges of Surgeons of Edinburgh and Ireland. 2010;8(3):144-50. 
39. Adelani MA, Harris AHS, Bowe TR, Giori NJ. Arthroscopy for Knee Osteoarthritis Has Not Decreased After a Clinical Trial. Clin Orthop Relat Res. 2016;474(2):489-94.

40. Harris IA, Madan NS, Naylor JM, Chong S, Mittal R, Jalaludin BB. Trends in knee arthroscopy and subsequent arthroplasty in an Australian population: a retrospective cohort study. BMC Musculoskelet Disord. 2013;14:143.

41. Zikria B, Hafezi-Nejad N, Wilckens J, Ficke JR, Demehri S. Determinants of knee replacement in subjects with a history of arthroscopy: data from the osteoarthritis initiative. European journal of orthopaedic surgery \& traumatology : orthopedie traumatologie. 2016;26(6):665-70.

Submit your next manuscript to BioMed Central and we will help you at every step:

- We accept pre-submission inquiries

- Our selector tool helps you to find the most relevant journal

- We provide round the clock customer support

- Convenient online submission

- Thorough peer review

- Inclusion in PubMed and all major indexing services

- Maximum visibility for your research

Submit your manuscript at www.biomedcentral.com/submit
Biomed Central 